\title{
A New Standstill Position Detection Technique for Nonsalient Permanent-Magnet Synchronous Motors Using the Magnetic Anisotropy Method
}

\author{
Jan Persson, Miroslav Markovic, and Yves Perriard
}

Integrated Actuators Laboratory (LAI), Ecole Polytechnique Fédérale de Lausanne (EPFL), CH-1015 Lausanne, Switzerland

\begin{abstract}
This paper describes a new method to estimate the standstill position of permanent-magnet synchronous motors (PMSMs). The method is based on the anisotropic properties of permanent magnets and is therefore referred to as a magnetic anisotropy method (MAM). It differs significantly from all standard standstill position detection methods previously presented in the literature. It is totally independent of saliency and saturation effects and can therefore be used in many types of PMSMs where the standard methods would fail. The paper fully explains the experimental results and briefly analyzes the physical phenomena behind the method.
\end{abstract}

Index Terms-Magnetic anisotropy method (MAM), permanent-magnet synchronous motor (PMSM), sensorless control, standstill position detection.

\section{NOMENCLATURE}

$\begin{array}{ll}B & \text { Magnetic flux density [T]. } \\ H & \text { Magnetic field intensity [A/m]. } \\ i & \text { Phase current [A]. } \\ L & \text { Phase inductance [H]. } \\ n_{s} & \text { Number of loops in stator winding [-]. } \\ S & \text { Surface [m²]. } \\ u_{i} & \text { Induced voltage in third phase [V]. } \\ \Delta u_{i} & \text { Differential } u_{i}[\mathrm{~V}] . \\ \arg \left(u_{i}\right) & \text { Phase shift of } u_{i}[\mathrm{deg}] . \\ \hat{u}_{i} & \text { Amplitude of } u_{i}[\mathrm{~V}] . \\ \hat{u}_{i a, b, c} & \text { Induced voltage in phase A,B,C [V]. } \\ u_{s} & \text { Voltage from voltage source [V]. } \\ u_{\alpha, \beta} & \text { Voltage after Clarke transf. [V]. } \\ x & \text { Linear rotor position [mm]. } \\ \theta & \text { Angular rotor position [deg]. } \\ \mu_{0} & \text { Permeability [Vs/Am]. } \\ \mu_{r} & \text { Relative permeability [-]. } \\ \mu_{r e} & \text { Easy axis relative permeability [-]. } \\ \mu_{r h} & \text { Hard axis relative permeability [-]. } \\ \Psi_{a, b, c} & \text { Magnetic flux in phase A,B,C [T]. } \\ \omega_{i} & \text { Injected signal frequency [rad/s]. }\end{array}$

\section{INTRODUCTION}

CENSORLESS control of permanent-magnet synchronous motors (PMSMs) is quickly becoming a popular alternative to bulky and expensive position sensors. Most sensorless control methods are based on the back-EMF voltage; see [1]-[5].

Digital Object Identifier 10.1109/TMAG.2006.887854

Color version of Fig. 1 is available online at http://ieeexplore.ieee.org.
All these methods, however, show severe limitations in the low speed region. To overcome this problem, several authors have proposed the use of high frequency or pulsewidth modulation (PWM) signals to track saturation and/or saliency effects. A voltage carrier signal can for example be injected via the PWM inverter. By measuring the phase currents, saliency information can be extracted by tracking the orientation of the ellipse that is given by a time varying $d$ - and $q$-axis current; see [6] and [7]. Others have proposed more complex tracking observers [8]-[10] to achieve a similar result.

In [11], it is described how the high frequency content in the phase currents can be used to extract the rotor position for a PMSM motor. The method relies upon several current measurements during one PWM period. To arrive at a solution, the PWM signal has to have some special properties which are not guaranteed by a standard space-vector PWM. A modified PWM algorithm is therefore also defined. The algorithm shows good results over a large speed range, including zero speed. The modified PWM algorithm, however, induces additional core losses into the motor. In contrast to the previous method, the authors of [12] propose the analyzation of the PWM voltages instead of the phase currents in a squirrel cage induction motor. This requires some extra hardware and analog filtering, and the method also needs access to the motor's neutral point. The method shows a thorough analysis of the high frequency components in the measured signal. This includes an analysis of the propagation of traveling waves on the motor cables as well as the influence of high frequency common-mode currents. Many of these methods have shown promising results all the way down to zero speed, but these techniques work only for motors which are either highly saturated or which have a pronounced spatial saliency. For all other motor types, there exist until today no solution for determining the rotor position at low speed and standstill.

This paper presents a new method to estimate the standstill rotor position for PMSMs in which the saturation and saliency 

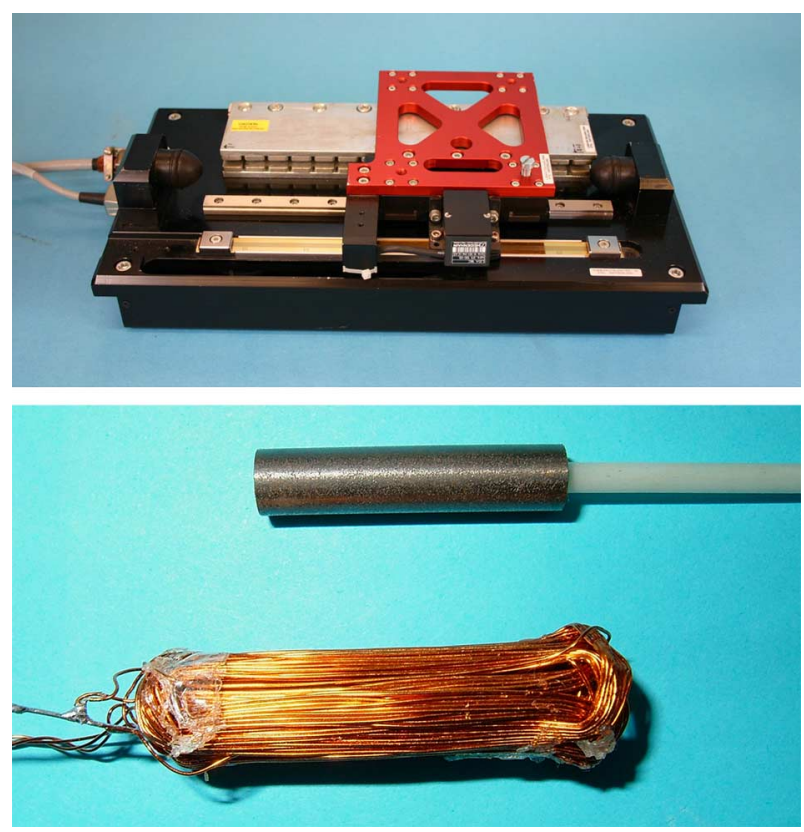

Fig. 1. Upper: Linear motor. Lower: Stripped rotational motor.

effects are negligible or nonexistent. The method is based on the anisotropic properties of permanent magnets and is therefore referred to as the magnetic anisotropy method or MAM $^{1}$ [13]. The MAM differs significantly from all standard standstill position detection methods previously presented in the literature. The proposed method is independent of any ferromagnetic material and can therefore be used in many types of PMSMs where the standard methods would fail. The method injects a very high frequency signal to detect anisotropic properties of the permanent magnets. When other authors claim to use high frequency signals, it is usually in the range of $0.5-3 \mathrm{kHz}$. The MAM has a significantly higher signal bandwidth of $100-500 \mathrm{kHz}$. The phenomenon is however very frequency dependent and the excitation frequency should be carefully chosen. A short overview of the measurement setup is presented and the experimental results are fully explained. Since the method is new and still under research, the priority has been given to experiments which can help to explain and understand this phenomena.

\section{MeAsurement SETUP}

To demonstrate the versatility of the proposed MAM, two completely different motor types are used. The first motor is a linear ironless motor with SmCo magnets, and the second is a "stripped" rotational motor with $\mathrm{NdFeB}$ magnets; see Fig. 1. The MAM can be used with both motors. However, the linear motor is equipped with a position sensor and the experimental results in Section III are obtained using this motor. The rotational motor is much simpler, and is used to analyze and explain the phenomena as shown in Section IV.

Both the linear and the rotational motor have three-phase, Y-connected windings. For the rotational motor with an accessible neutral point $\mathrm{N}$, the measurements are made according to the setup in Fig. 2. For the linear motor, the neutral point is

\footnotetext{
${ }^{1}$ Patent pending: EPO No. EP05102754.8.
}

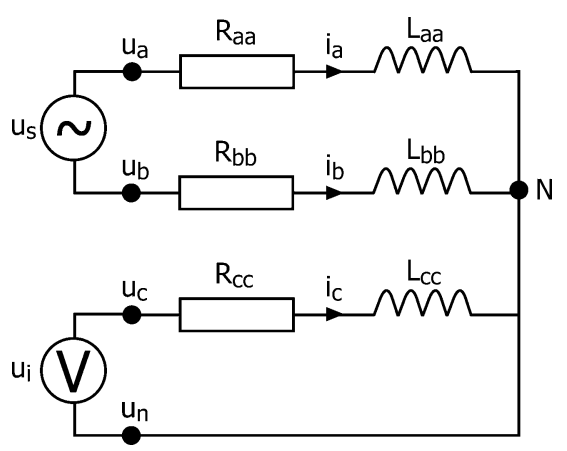

Fig. 2. Measurement setup with neutral point accesible.

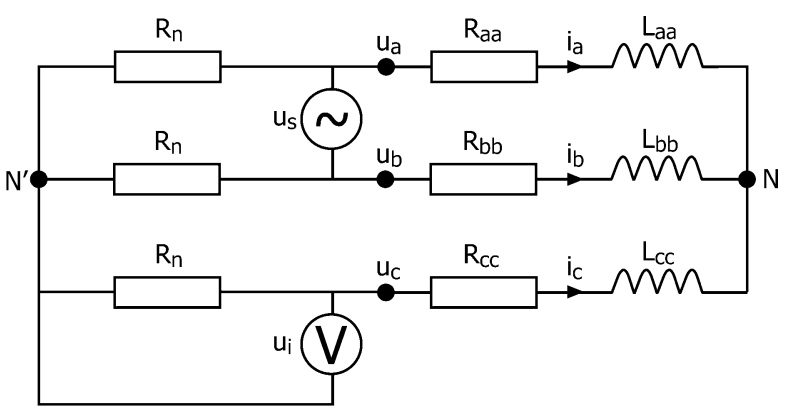

Fig. 3. Measurement setup with artificial neutral point.

not accessible and the measurements are made with an artificial neutral point N' as shown in Fig. 3. For both motors the measured voltage $u_{i}$ is referenced to $\mathrm{N}$ or $\mathrm{N}^{\prime}$, and this point cannot be connected to ground without disturbing the measurement. Therefore, it is necessary to use a differential amplifier for all measurements of $u_{i}$. The following description is valid for linear as well as rotational motors.

The measurements are made by connecting a sinusoidal signal generator with a voltage $u_{s}$ between phase $\mathrm{A}$ and $\mathrm{B}$ in the three phase windings; see Figs. 2 and 3. The voltage amplitude is adjusted to give a phase current of $10 \mathrm{~mA}$. The frequency of the voltage signal depends on the application, but it is chosen in the range 100-500 kHz. In Section III, a detailed discussion on the choice of an appropriate frequency is presented.

The current in phase $\mathrm{A}$ and $\mathrm{B}$ will induce a voltage $u_{i}$ in the third phase C. This voltage will of course have the same frequency and shape as the excitation current, but there is also a position-dependent component that modifies the amplitude $\hat{u}_{i}$ and the phase $\arg \left(u_{i}\right)$ of the measured voltage. The definitions of $\hat{u}_{i}$ and $\arg \left(u_{i}\right)$ are illustrated in Fig. 4. In this figure, the voltage $u_{i}$ at six different rotor positions is shown. The frequency of the test signal is approximately $100 \mathrm{kHz}$. This demonstrates a typical behavior of $u_{i}$ when the rotor position $\theta$ changes approximately 45 electrical degrees. In Fig. $4, u_{i}$ is presented in p.u. units since the true voltage level is of little importance.

To inject the signal at the frequency of $100-500 \mathrm{kHz}$, a voltage source is used, with a referential sinusoidal signal obtained from a signal generator. For the industrial application of the method, special electronics should be used (e.g., an additional chip capable of generating such a high frequency). 


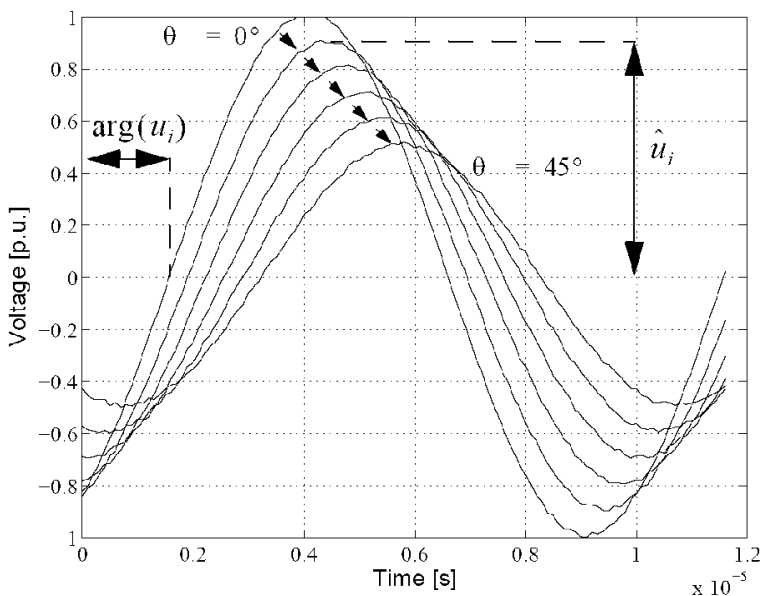

Fig. 4. The amplitude and the phase of $u_{i}$ change with the rotor position as $\theta$ changes between $0^{\circ}$ and $45^{\circ}$.

\section{EXPERIMENTAL RESULTS}

For the experimental results in this section, only the linear motor is used since it is equipped with a high precision position sensor. At first, the raw measurements of the position-dependent variable $\hat{u}_{i}$ are presented. Thereafter, it is shown how these measurements can be used to estimate the rotor position. Finally, it is also discussed how the frequency of the sinusoidal voltage signal can be chosen in an optimal way.

\section{A. Raw Measurements of the MAM}

The position dependency of the measured signal $\hat{u}_{i}$ becomes evident when the measurement results in Fig. 5 are considered. This figure shows how the amplitude $\hat{u}_{i}$ changes as the linear motor make a movement of approximately $100 \mathrm{~mm}$. This movement at a slow constant speed $(\sim 1 \mathrm{~mm} / \mathrm{s})$ is imposed externally. A high-pass filter with a cutoff frequency around $100 \mathrm{~Hz}$ is used to eliminate any induced voltages created by the rotor movement. One full electrical period equals a linear movement of $32 \mathrm{~mm}$, so it can be noticed that the periodicity of the measured signal is twice the electrical periodicity.

From Fig. 5, it can be seen that the measured signal is nonsymmetrical. For the linear motor, this can be partly explained by end effects due to the mechanical design of this motor. The same nonsymmetrical behavior can however also be seen in the rotational motor and in this case the explanation can be found in the anisotropy of the magnet. The anisotropy effects in rare-earth magnets are discussed further in Section IV.

\section{B. Rotor Position Estimation}

All measurements for the linear motor are made with an artificial neutral point as shown in Fig. 3. To remove the offset that can be seen in Fig. 5, a second measurement for the same phase is made but with the voltage source terminals inverted. It should be noted that the voltage source produces a sinusoidal voltage between 0 and $12 \mathrm{~V}$ with a positive offset of $6 \mathrm{~V}$. A resistor is also connected in series with the motor phase terminal to reduce the direct current.

The measurement is thus repeated twice, which results in a "positive" measurement $\hat{u}_{i+}$ and a "negative" measurement $\hat{u}_{i-}$

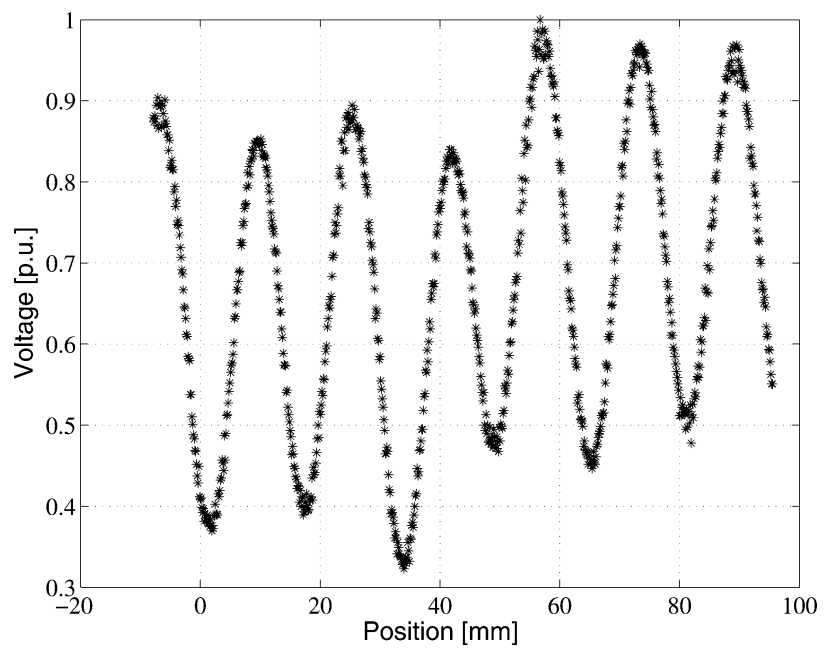

Fig. 5. Change in amplitude $\hat{u}_{i}$ when the linear motor moves $100 \mathrm{~mm}$ at a low constant speed.

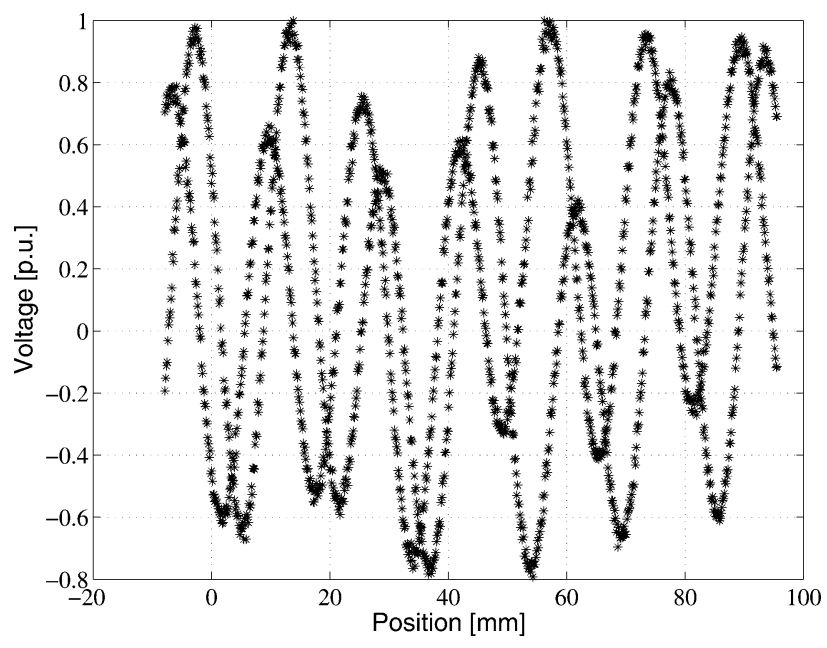

Fig. 6. Position-dependent variables $\hat{\mu}_{\alpha}$ and $\hat{\mu}_{\beta}$.

for the same phase. The measurements are repeated for the other two phases which gives a total of six measurements. The difference between each pair of $+/-$ measurements are then calculated as

$$
\begin{aligned}
\hat{\mu}_{i a} & =\hat{\mu}_{i a+}-\hat{\mu}_{i a-} \\
\hat{\mu}_{i b} & =\hat{\mu}_{i b+}-\hat{\mu}_{i b-} \\
\hat{\mu}_{i c} & =\hat{\mu}_{i c+}-\hat{\mu}_{i c-} .
\end{aligned}
$$

These three variables are then reduced to two $\left(\hat{\mu}_{\alpha}\right.$ and $\left.\hat{\mu}_{\beta}\right)$ by using the Clark transformation. The result is two more or less sinusoidal signals, which can be studied in Fig. 6 .

From the data points in Fig. 6, two reference signals $\hat{\mu}_{\alpha \text { ref }}$ and $\hat{\mu}_{\beta \text { ref }}$ are created. Each reference signal contains $n=50$ data points. Given these reference signals, the position from a new measurement $\hat{\mu}_{\alpha}$ and $\hat{\mu}_{\beta}$ is calculated by choosing the rotor position $x_{k}$ that minimizes the expression

$$
\min _{x_{k}}\left(\left|\hat{\mu}_{\alpha}-\hat{\mu}_{\alpha \mathrm{ref}}\left(x_{k}\right)\right|+\left|\hat{\mu}_{\beta}-\hat{\mu}_{\beta \mathrm{ref}}\left(x_{k}\right)\right|\right) \quad k=1 \ldots n \text {. }
$$



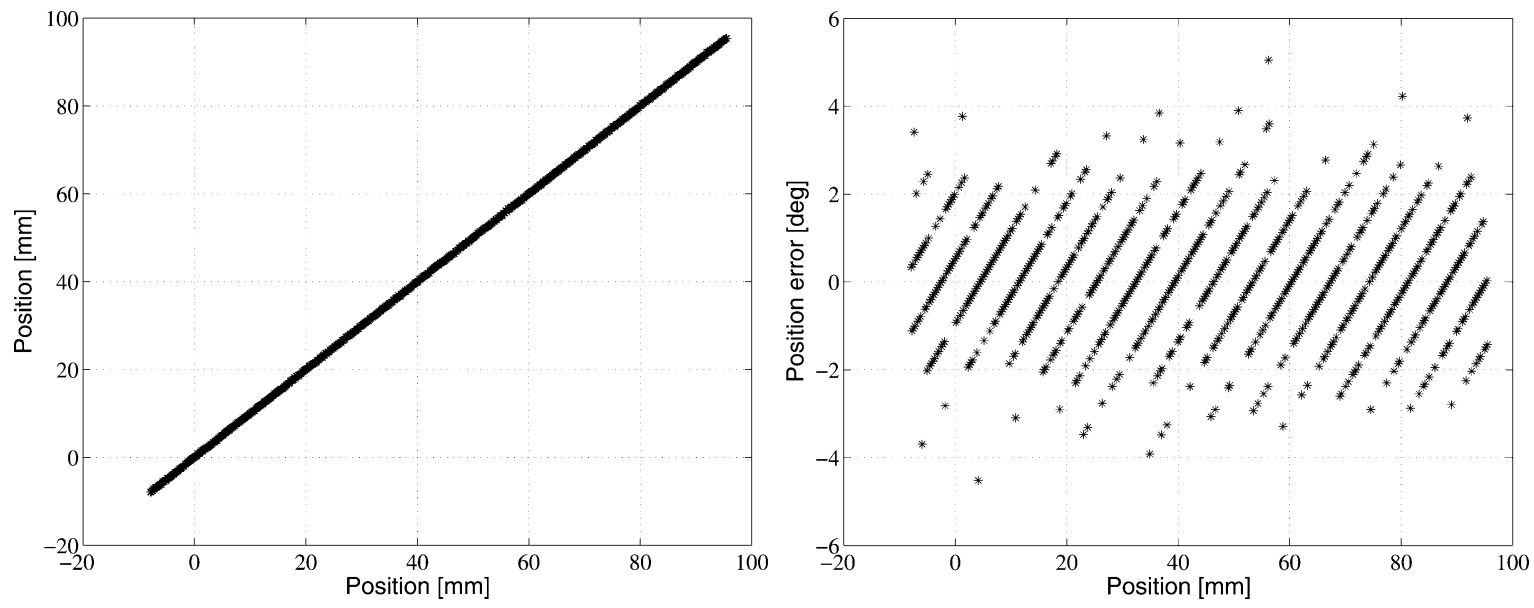

Fig. 7. Left: Position estimation versus True position. Right: Position error versus True position.

TABLE I

POSITION ESTIMATION ERRORS

\begin{tabular}{|l|c|c|}
\hline \multicolumn{3}{|c|}{ Results of MAM (Fig. 7) } \\
\hline Mean error & $\mu$ & $0.084^{\mathbf{o}}$ \\
\hline Standard deviation & $\sigma_{s}$ & $1.4^{\mathrm{o}}$ \\
\hline Maximum error & $\varepsilon_{\max }$ & $5.1^{\mathrm{o}}$ \\
\hline
\end{tabular}

The experimental results presented in this section are primarily based on the amplitude difference $\hat{u}_{i}$. This choice has been made since it is easier to measure amplitude rather than phase difference with a good precision. The phase difference $\arg \left(u_{i}\right)$ could also be used as information source, but so far nothing indicates that this could improve the precision of the MAM.

Using (2) on a second data set gives the results in Fig. 7. Note that the data that was used to calculate the reference points are different from those used in the estimation of the rotor position. The position estimation error is shown in the right part of Fig. 7 and the mean error, the standard deviation, and the maximum error are presented in Table I. The standard deviation of the estimation error is as small as $1.4^{\circ}$ and the maximum error is $5.1^{\circ}$. This precision is more that sufficient to insure a correct operation of the motor. A quantization error due to the equidistant reference points can be seen in Fig. 7; this is however inevitable and the precision cannot be improved by using more reference points. This has been tested by introducing more reference points, but despite this the standard deviation of the position estimation error did not decrease. The only possible way to get better results would be to improve the signal-to-noise ratio of the measured signal.

A full stroke of the linear motor corresponds to approximately 3.5 electrical periods. In Fig. 7, errors which are a multiple of $180^{\circ}$ electrical degrees have been corrected. This can be compared to a tracking observer where the position is surveyed in permanence.

Due to the fact that the variables $\hat{\mu}_{\alpha}$ and $\hat{\mu}_{\beta}$ in Fig. 6 are nonsymmetrical and irregular, it was possible to correctly determine the mechanical position $x$ in $68.5 \%$ of the cases. For this experiment, a third data set was taken from the linear motor and these results can be studied in Fig. 8. Other standstill methods based on for example inductance saliency can usually not be used to determine the mechanical position. Even though these first results of the MAM do not show $100 \%$ accuracy, it is interesting to see that in some cases the mechanical and not only the electrical position can be determined. The maximum error and standard deviation are not presented in this case since they make no sense when the errors are multiples of 180 electrical degrees.

\section{Choosing an Optimal Frequency}

Measurements have shown that the observed phenomenon is very frequency dependent. In order to have a good result, it is of great importance to carefully choose the frequency $\omega_{i}$ of the excitation signal. To do this, numerous measurements have been made at different frequencies. The result is presented in Fig. 9. This graph shows the frequency dependency of the measured voltage $\hat{u}_{i}$. The vertical axis represents the difference $\Delta \hat{u}_{i}$ between the maximum and the minimum value of $\hat{u}_{i}$ for one full stroke, i.e.,

$$
\Delta \hat{\mu}_{i}=\max \left(\hat{\mu}_{i}(x)\right)-\min \left(\hat{\mu}_{i}(x)\right) \quad x \in[0,100] \mathrm{mm} .
$$

It can be seen that there is a maximum around $350 \mathrm{kHz}$, while $\Delta \hat{u}_{i}$ quickly approaches zero when the frequency is under $20 \mathrm{kHz}$. Higher frequencies make $\Delta \hat{u}_{i}$ smaller. To have a maximum precision of the algorithm, the excitation frequency for this motor should therefore be chosen around $350 \mathrm{kHz}$.

\section{Explaining the Phenomena}

So far the results of the MAM have been presented. This section will focus on explaining the phenomena that has been observed. Since the mechanical structure of the linear motor is quite complex, the attention is turned to the rotational motor in Fig. 1 which has a much simpler configuration. This "stripped" rotational motor consists of one free-standing stator winding, and a rotor made of a diametrically magnetized $\mathrm{NdFeB}$ magnet. Furthermore, the phenomenon has been observed with a magnetized as well as a nonmagnetized magnet. All measurement results that were presented in Section III can be repeated also for the rotational motor, but for space considerations these results are not presented in this paper. 

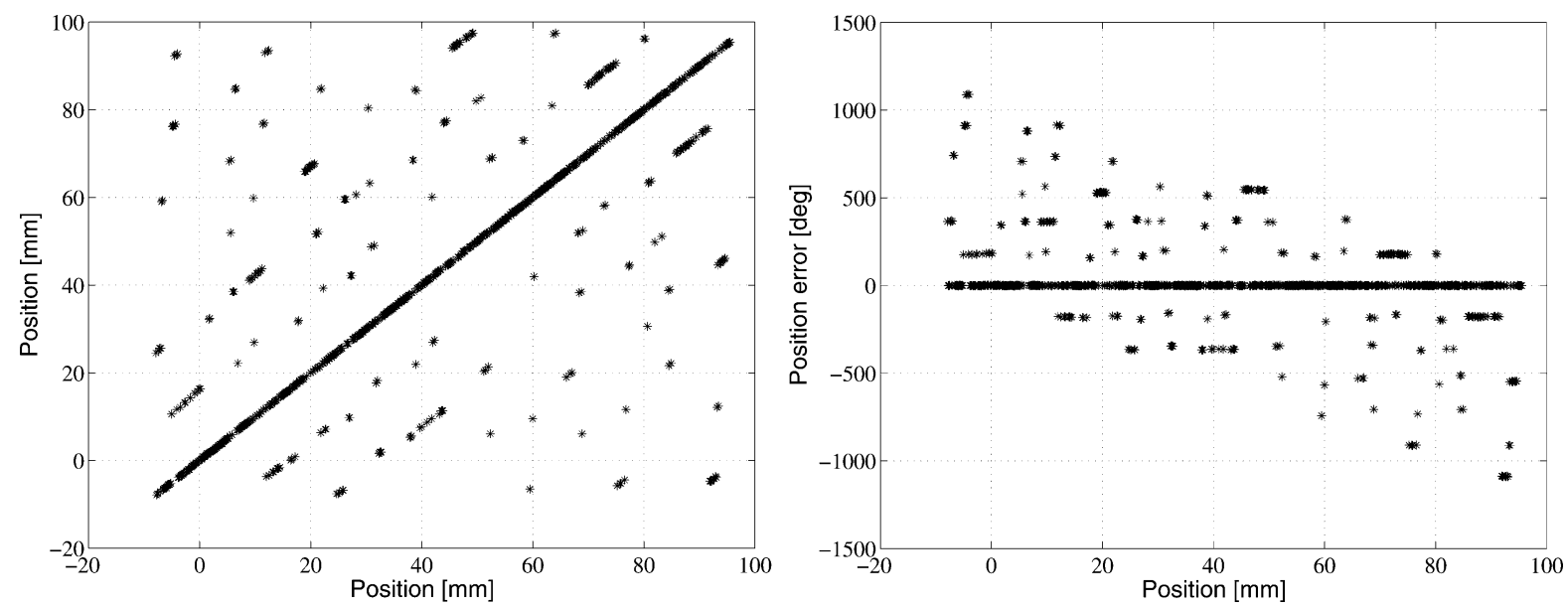

Fig. 8. In $68.5 \%$ of the cases it is possible to correctly estimate the mechanical position $x$ (Left: Position estimation versus True position. Right: Position error versus True position.).

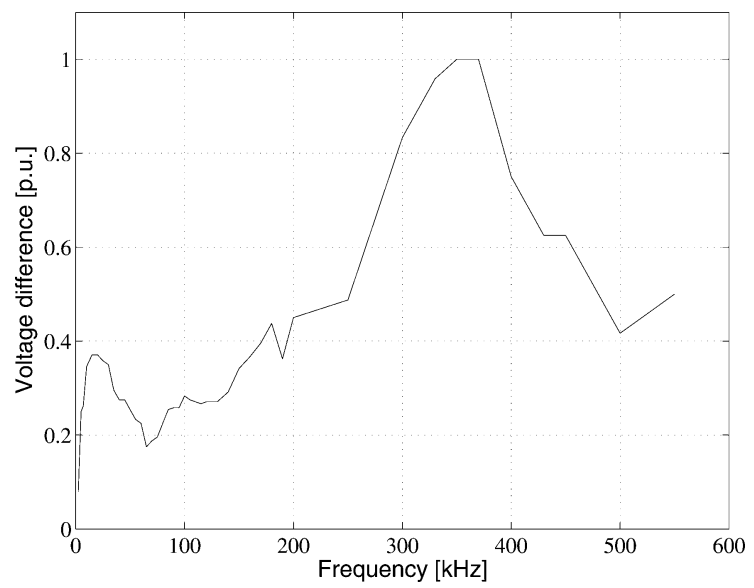

Fig. 9. Frequency dependency of $\Delta \hat{u}_{i}$.

\section{A. Motor Symmetry and Magentic Flux}

When this phenomenon was observed for the first time, one of the most surprising discoveries was the large change in the measured voltage. For the rotational test motor, amplitude changes of up to $100 \%$ with a phase shift of $180^{\circ}$ have been observed. From this observation a first, but incautious conclusion could be that this is a very distinct phenomenon capable of significant changes in the flux paths. This is however not correct. The phenomenon becomes visible due to the symmetry, or rather the small asymmetry, that exists between the three phase windings. Consider the simplified model of a three phase rotational motor in Fig. 10. The current $i$ in phase A generates the flux vector $\Psi_{a}$, and the negative current $-i$ in phase B generates the flux vector $\Psi_{b}$. The flux $\Psi_{c}$ in the third phase $\mathrm{C}$ is the vector sum of $\Psi_{a}$ and $\Psi_{b}$. In Fig. 10, it is demonstrated how the flux $\Psi_{c}$ changes at four different rotor positions $\theta$ between $0^{\circ}-90^{\circ}$ electrical degrees. It can be seen how the flux $\Psi_{c}$ becomes negative due to only small changes in the flux vectors $\Psi_{a}$ and $\Psi_{b}$. If these vectors change with only $1 \%-2 \%$, this will give a visible change in $\Psi_{\mathrm{C}}$.

This can also been shown mathematically if it is assumed that the phase inductance have one constant part $L_{a a}, L_{b b}, L_{c c}$ and

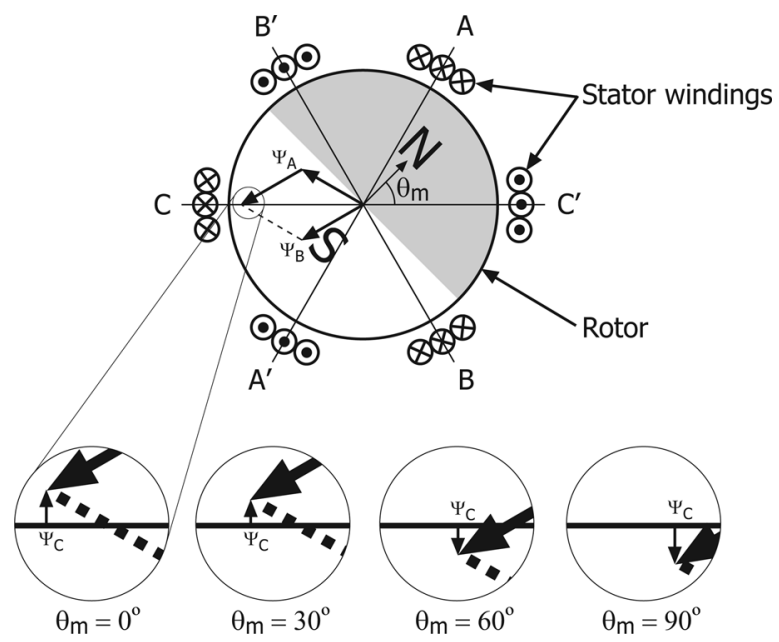

Fig. 10. Imbalance in the stator windings gives an induced voltage in the nonsupplied phase.

one position-dependent part $L_{e}$. The inductance $L_{e}$ is the result of a magnetic anisotropy phenomenon as shown in Section B. Thus, the magnetic flux in phase A and B can be written as

$$
\begin{aligned}
& \psi_{a}=L_{a} i_{a}=\left(L_{a a}+L_{e} \cos (2 \theta)\right) \hat{i} \sin \left(\omega_{i} t\right) \\
& \psi_{b}=L_{b} i_{b}=-\left(L_{b b}+L_{e} \cos \left(2 \theta-\frac{2 \pi}{3}\right)\right) \hat{i} \sin \left(\omega_{i} t\right) .
\end{aligned}
$$

From this equation, the flux $\Psi_{c}$ in the third phase can be found as

$$
\psi_{c}=\frac{\psi_{a}}{2}-\frac{\psi_{b}}{2} .
$$

By deriving this expression, the amplitude of the induced voltage $\hat{u}_{i c}$ in phase $\mathrm{C}$ can be found as

$$
\hat{\mu}_{i c}=\frac{\hat{i} \omega_{i}}{2}\left(L_{a a}-L_{b b}+\sqrt{3} L_{e} \cos \left(2 \theta+\frac{\pi}{6}\right)\right) .
$$




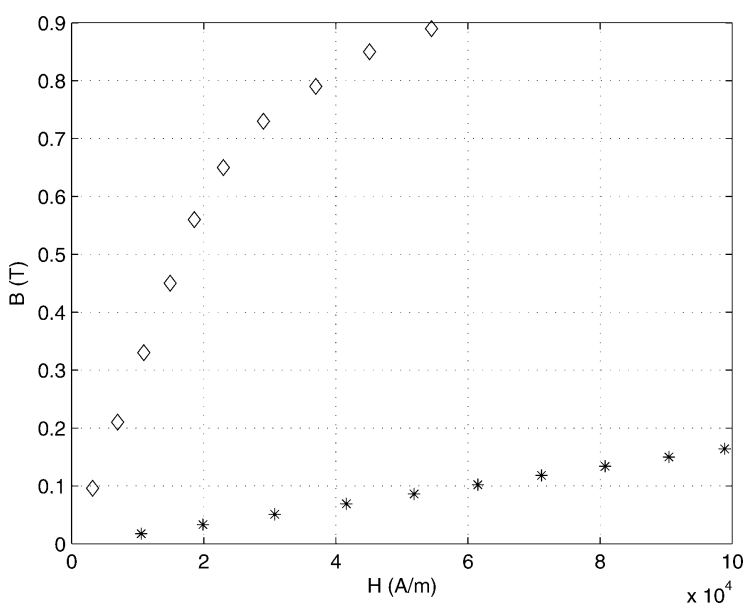

Fig. 11. Hard (*) and easy $(\diamond)$ axis magnetization curve for $\mathrm{NdFeB}$.

This expression corresponds well to what has been observed for the linear motor in Section III. For the rotational motor, it was previously mentioned that amplitude changes up to $100 \%$ have been witnessed. This can be explained if the symmetry of the motor is very good; this means that $L_{a a}=L_{b b}$ and (6) can be reduced to

$$
\hat{\mu}_{i c}=\frac{\sqrt{3} \hat{i} \omega_{i} L_{e}}{2} \cos \left(2 \theta+\frac{\pi}{6}\right)
$$

This shows that for motors with a good symmetry, large changes in $\hat{u}_{i c}$ are possible.

\section{B. Anisotropy Phenomenon}

Now we turn the attention to the physical phenomena behind the proposed method. Modern rare earth magnets such as SmCo or $\mathrm{NdFeB}$ are intentionally made anisotropic as a result of the production process. The dipole elements in the magnet will have a predisposition to align themselves in one direction called the easy axis. Thus, the magnetization vector can be separated into two components: the easy axis component and a component perpendicular to this one called the hard axis [14].

There is actually another hard axis which is axial, but this axis is not important since it is perpendicular to the main magnetic flux. It is well known that the magnetization curve in the hard axis is significantly different from the magnetization curve in the easy axis; see for example [15]-[17]. In Fig. 11, the magnetization curve for the hard axis as well as the easy axis is shown for a sample of a nonmagnetized $\mathrm{NdFeB}(\mathrm{N} 40 \mathrm{H})$ magnet. The nonsaturated permeability in the easy axis is $\hat{\mu}_{r e}=24$ and in the hard axis $\mu_{r h}=1.3$.

In [18], it is also shown how the magnetic field in a PMSM can be decomposed into two vectors: one for the easy axis $H_{e}$ and one for the hard axis $H_{\mathrm{h}}$. The total magnetic flux density $B$ can then be calculated by using two magnetization curves, one for each axis. It is given from Fig. 11 that each magnetization curve has a different relative permeability $\hat{\mu}_{r e}$ and $\hat{\mu}_{r h}$; see also [15]. From these results, it is easy to realize that the magnetic flux $\Psi_{a}$ and $\Psi_{b}$ in the test motor are influenced by the orientation of the easy axis since

$$
\Psi=n_{s} \int_{S} \mu_{0} \mu_{r} H d S
$$

where $n_{s}$ is the number of turns in the stator winding, $H$ the magnetic field intensity and $S$ the total surface. As $\mu_{r}$ change, there will also be changes in $\Psi$ which can be interpreted as the position-dependent inductance $L_{e}$ introduced in (4). From the discussion above, it is possible to draw the conclusion that a variable relative permeability caused by the anisotropic properties of rare-earth magnets is the most likely candidate when it comes to explaining the observed phenomena. Remember that it is enough that the flux vectors $\Psi_{a}$ and $\Psi_{b}$ change $1 \%-2 \%$ before the phenomenon becomes visible to the MAM.

\section{CONCLUSION}

A new method to determine the rotor position at standstill has been proposed. The method is called a magnetic anisotropy method (MAM) since it is based on anisotropic properties of modern rare-earth magnets. The method has been tested on $\mathrm{SmCo}$ and $\mathrm{NdFeB}$ magnets, but it most likely works also for other types of anisotropic magnets such as Alnico. The method uses an excitation signal in the range of $100-500 \mathrm{kHz}$. The optimal frequency depends however strongly on the magnet type. The proposed method works also for nonsaturated, nonsaliency motors. This is a motor type for which all standard standstill position detection methods would fail. Furthermore, it is possible to detect the position for a nonmagnetized rotor. This might be rare in PMSM, but this feature could be used for example in the production process of permanent magnets to determine magnet orientation.

The MAM is new and has not previously been referenced in the literature; therefore, there are several areas still open for research. First, the influence of the temperature should be compensated: as the PM properties change with the temperature, the method should take these changes into account. The second important issue is how to apply the MAM at high speeds, as the PWM harmonics should be filtered out from the voltage $u_{i}$. Finally, an adequate hardware for both generation of the signal $u_{s}$ and measurement of the signal $u_{i}$ should be realized in order to industrialize the MAM.

\section{REFERENCES}

[1] S. Bolognani, M. Zigliotti, and M. Zordan, "Extended-range PMSM sensorless speed drive based on stochastic filtering," IEEE Trans. Power Electron., vol. 16, no. 1, pp. 110-117, Jan. 2001.

[2] B.-J. Brunsbach, G. Henneberger, and T. Klepsch, "Position controlled permanent excited synchronous motor without mechanical sensors," in Fifth Eur. Conf. Power Electronics and Applications, Brighton, U.K., Sep. 1993.

[3] J. C. Moreira, "Indirect sensing for rotor flux position of permanent magnet AC motors operating over a wide speed range," IEEE Trans. Ind. Appl., vol. 32, no. 6, pp. 1394-1401, Dec. 1996.

[4] J. X. Shen, Z. Q. Zhu, and D. Howe, "Improved speed estimation in sensorless PM brushless AC drives," IEEE Trans. Ind. Appl., vol. 38, no. 4, pp. 1072-1080, Jul.-Aug. 2002. 
[5] Q. Jiang, C. Bi, and R. Huang, "A new phase-delay-free method to detect back EMF zero-crossing points for sensorless control of spindle motors," IEEE Trans. Magn., vol. 41, no. 7, pp. 2287-2294, Jul. 2005.

[6] T. Noguchi and S. Kohno, "Mechanical-sensorless permanent-magnet motor drive using relative phase information of haramonic currents caused by frequency- modulated three phase PWM carriers," IEEE Trans. Ind. Appl., vol. 39, no. 4, pp. 1085-1092, Jul.-Aug. 2003.

[7] M. Schroedl, "Detection of the rotor position of a permanent magnet synchronous machine at standstill," in Proc. Int. Conf. Electrical Machines, ICEM '88, Pisa, Italy, Sep. 1988.

[8] F. Briz, W. M. Degner, P. Garcia, and R. D. Lorenz, "Comparison of salincy-based sensorless control techniques for AC machines," IEEE Trans. Ind. Appl., vol. 40, no. 4, pp. 1107-1115, Jul.-Aug. 2003.

[9] H. Kim, K.-K. Huh, R. D. Lorenz, and M. T. Jahns, "A novel method for initial rotor position estimation for IPM synchronous machine drives," IEEE Trans. Ind. Appl., vol. 40, no. 5, pp. 1369-1378, Sep.-Oct. 2004.

[10] S. Shinnika, "New "Mirror-phase vector control" for sensorless drive of permanent magnet-magnet synchronous motor with pole saliency," IEEE Trans. Ind. Appl., vol. 40, no. 2, pp. 599-606, Mar./Apr. 2004.

[11] V. Petrovic, A. M. Stankovic, and V. Blasko, "Position estimation in salient PM synchronous motors based on PWM excitation transients," IEEE Trans. Ind. Appl., vol. 39, no. 3, pp. 835-843, May-Jun. 2003.

[12] J. Holz and H. Pan, "Acquisition of rotor anisotropy signals in sensorless position control systems," IEEE Trans. Ind. Appl., vol. 40, no. 5, pp. 1379-1387, Sep.-Oct. 2004.
[13] J. Persson, "Innovative Standstill Position Detection Combined with Sensorless Control of Synchronous Motors," Thesis No. 3221, EPFL, Lausanne, Switzerland, 2005.

[14] F. Deng, "An improved iron loss estimation for permanent magnet brushless machines," IEEE Trans. Energy Convers., vol. 14, no. 4, pp. 1391-1395, Dec. 1999.

[15] P. Campbell, "A model of anisotropic alnico magnets for field computation," IEEE Trans. Magn., vol. MAG-18, no. 3, pp. 898-904, May 1982.

[16] P. Campbell and S. A. Al-Murshid, "The effect of magnetization distribution within anisotropic alnico magnets upon field calculation," IEEE Trans. Magn., vol. MAG-16, no. 5, pp. 1032-1034, Sep. 1980.

[17] M. Enokizino, "Magnetic field analysis of permanent magnet motor with magneto anisotropic material Nd-Fe-B," IEEE Trans. Magn., vol. 39, no. 3, pp. 1373-1376, May 2003.

[18] J. Slomczynska, "No-load magnetic-field distribution in a permanent magnet electrical machine," IEEE Trans. Magn., vol. MAG-11, no. 6, pp. 1720-1722, Nov. 1975.

Manuscript received September 12, 2006; revised November 2, 2006. Corresponding author: Y. Perriard (e-mail: yves.perriard@epfl.ch). 